Sharif University of Technology
Scientia Iranica
Transactions E: Industrial Engineering
hCIENTIA

\title{
A novel fuzzy multi-objective method for supplier selection and order allocation problem using NSGA II
}

\author{
M.A. Sobhanolahi, A. Mahmoodzadeh*, and B. Naderi \\ Department of Industrial Engineering, Faculty of Engineering, Kharazmi University, Tehran, Iran.
}

Received 21 February 2018; received in revised form 27 July 2018; accepted 15 October 2018

\section{KEYWORDS}

Supplier selection;

Order allocation;

Fuzzy theory;

Multi-objective

programing;

NSGA II.

\begin{abstract}
This paper introduces a supplier selection and order allocation problem in a single-buyer-multi-supplier supply chain in which appropriate suppliers are selected and orders allocated to them. Transportation costs, quantity discount, fuzzy-type uncertainty, and some practical constraints were taken into account in the problem. The problem was formulated as a bi-objective model to minimize annual supply chain costs and to maximize Annual Purchasing Value $(A P V)$. The fuzzy weights of suppliers, which were the output of one of the supplier evaluation methods, were considered in the second objective function. Then, a novel fuzzy multi-objective programming method was formulated for obtaining Pareto solutions. The method is the extension of a single-objective method existing in the literature. It is based on the degree of satisfaction of the Decision Maker (DM) with each fuzzy objective considering the fulfillment level of fuzzy constraints. In the proposed method, the problem remains multi-objective and, unlike in the existing methods, it is not transformed into a single-objective model. At the last stage of the proposed method, the fuzzy results are compared with an index and the DM can identify the appropriate or inappropriate solutions. To solve the problem, Non-dominated Sorting Genetic Algorithm (NSGA II) is designed and computational results are presented using numerical examples.

(C) 2020 Sharif University of Technology. All rights reserved.
\end{abstract}

\section{Introduction}

In the current competitive environment, supplier evaluation and selection is one of the most important processes in supply chain management for any organization. It is critical since suppliers have a major impact on strategic and operational performance of organizations. Also, this process plays an important role in determining the cost, quality, and other aspects of the finished product [1]. Hence, organizations rely

*. Corresponding author. Tel.: +982634551022 E-mail addresses: sobhanallahi@khu.ac.ir (M.A. Sobhanolahi); ahmad.mahmoodzade1@gmail.com (A Mahmoodzadeh); bahman.naderi@aut.ac.ir (B. Naderi)

doi: $10.24200 /$ sci. 2018.50484 .1717 more on suppliers to reduce their costs, to improve the quality of their products, or to focus on a specific part of their operations [2].

Supplier selection is complex since organizations must take into account multiple aspects including both quantitative and qualitative criteria $[3,4]$. In such cases, the criteria are conflicting and a trade-off among them is required. Therefore, selection of the best suppliers becomes a multi-criteria decision-making problem. Furthermore, the process becomes more complicated if parameters are incomplete or uncertain.

On the other hand, higher levels of inventory lead to increased supply chain responsiveness, but decrease cost efficiency because of inventory holding costs [5]. Inventory costs account for a number between 20 and $40 \%$ of the total product value. Hence, inventory management is one of the significant parts 
of supply chain management [6]. Allocating orders to the selected suppliers allows for some economies of scale through the right choice of quantities to order from each supplier. Sometimes, suppliers offer quantity discounts as a powerful incentive to motivate buyers to increase the amount of their ordered quantities [7]. Indeed, the unit price paid by buyers for large orders is usually smaller than the unit price of small orders [8]. Incremental quantity discounts, business volume quantity discounts, and all-unit quantity discounts are the three main types of quantity discounts [9].

This paper introduces a supplier selection and order allocation problem in a single-buyer-multi-supplier supply chain in which appropriate suppliers are selected and orders allocated to them. Transportation costs, quantity discount, fuzzy-type uncertainty, and some practical constraints are taken into account in the problem. Firstly, the problem is formulated as a biobjective model for minimizing annual supply chain costs and maximizing Annual Purchasing Value ( $A P V)$. The fuzzy weights of suppliers, which are the output of one of the supplier evaluation methods, are considered in the second objective function. The assumptions of the model are appropriate to the real-world conditions. Therefore, the model can be applied to any type of supply chain in which a buyer acquires the demanded items from some potential suppliers.

To overcome the complexity of the process, we propose a novel fuzzy multi-objective programming method for obtaining Pareto solutions. The method is an extension of the single-objective method proposed by Jiménez et al. [10]. It is based on the level of satisfaction of the Decision Maker (DM) with each fuzzy objective considering the degree of realization of fuzzy constraints. In the proposed method, the problem remains multi-objective and, unlike in the existing methods, it is not transformed into a single-objective model. At the last stage of the proposed method, the fuzzy results are compared with an index and the DM can identify the appropriate or inappropriate solutions. To solve the problem, Non-dominated Sorting Genetic Algorithm (NSGA II) is designed and computational results are presented using numerical examples.

The rest of this paper proceeds as follows. In Section 2, the related literature is briefly reviewed. In Section 3, we describe the problem, state the assumptions, and give the parameters, variables, and the formulation of the model. Section 4 deals with the novel fuzzy multi-objective programming method. Section 5 is devoted to describing the solution procedure, including changes in objectives and constraints, solution encoding, repair algorithm, and NSGA II procedure. In Section 6, we illustrate the findings of the implementation of the proposed methodology with some numerical examples. Finally, Section 7 concludes the study and presents future research directions.

\section{Literature review}

\subsection{Supplier selection and order allocation}

In this section, the researches which have been conducted in the area of supplier selection and order allocation with quantity discount are reviewed and their objectives and solution methods are addressed. Dahel [11] assumed multi-item volume discounts and proposed a multi-objective mixed-integer programming model, which could be solved through either a preference-oriented approach or the generating approach. Demirtas and Üstün [12] studied a model to maximize the purchasing value and to minimize the budget and the defect rates. They used analytical network process and the multi-objective mixed-integer programming, and adopted epsilon-constraint method and reservation level through Tchebycheff procedure to solve the problem. Xia and Wu [13] studied a problem to maximize total weighted quantity of purchasing, minimize total purchasing cost, minimize the number of defective items, and maximize the number of on-time delivered items. They proposed a two-stage method to solve the problem by means of Analytical Hierarchy Process (AHP) improved by rough set theory, at the first stage, and a multi-objective mixed-integer linear programming, at the second stage. Burke et al. [14] considered three types of discounts, namely linear quantity discount, incremental unit price discount, and all-unit quantity discount. They developed a heuristic to measure the effect of quantity discounts in the problem. Kokangul and Susuz [15] investigated a biobjective model by minimizing total purchasing cost and maximizing purchasing value obtained using AHP. They proposed a bi-objective non-linear programing model using goal programming. Amid et al. [16] studied a model which minimized total cost, the percentage of late-delivery items, and the percentage of rejected items. They developed a fuzzy multi-objective mixedinteger linear programming model to solve the problem. Wang and Yang [17] developed a model to minimize total cost, defective rate, and delivery lateness rate and proposed a two-stage procedure using AHP and a multi-objective mathematical programming. Ebrahim et al. [18] developed a model to minimize cost, late delivered items, and defective items considering three types of discount and proposed a Scatter Search (SS) algorithm and exact method to solve the problem. Razmi and Maghool [19] considered three types of discount in a fuzzy bi-objective model to minimize total purchasing cost and maximize total purchasing value. They adopted an augmented epsilon-constraint and reservation level by Tchebycheff models. Kamali et al. [20] investigated a model to minimize total annual cost, total number of defective items, and total number of late delivered items as well as to maximize total purchasing value. They proposed Particle Swarm Op- 
timization (PSO) and SS algorithm to solve the model. Zhang and Zhang [21] studied a single-objective model to minimize costs with stochastic demand and solved a mixed-integer programming in which all suppliers met the qualitative criteria level. Lee et al. [22] investigated a single-objective model to minimize total purchasing cost under all-unit and incremental quantity discounts and designed a genetic algorithm to solve the model. Pazhani et al. [5] addressed a single-objective model to minimize total cost per unit time considering the purchasing, setup, holding, and transportation costs. A mixed-integer nonlinear programming model was developed to solve the problem using exact methods. Moghaddam [23] set the four objectives of maximizing total net profit and minimizing total defective parts, total late deliveries, and total risk factors of the economic environment associated with each supplier. Bohner and Minner [24] considered both all-units and incremental quantity discounts as well as failure risk to minimize total costs and solved the mixed-integer linear programming model using an exact approach. Çebi and Otay [25] developed a model to minimize total cost, total late deliveries, and total defective items and to maximize total utility of the purchasing activity. Hamdan \& Cheaitou [1] considered green criteria to maximize total value of purchasing and minimize total costs. They proposed a three-stage method using fuzzy TOPSIS at the first stage to assign two preference weights to every potential supplier, AHP at the second stage to determine the importance weight of each supplier, and bi-objective integer linear programming at the third stage. The model was solved by the weighted comprehensive criterion method and the branch-andcut algorithm. Hamdan and Cheaitou [26] dealt with green criteria, quantity discounts, and varying supplier availability and applied the same three-stage method proposed in their previous study [1] in order to maximize total green and traditional values of purchasing and minimize total purchasing cost. Ranjbar Tezenji et al. [27] considered supplier location selection and order allocation under capacity constraints in a stochastic environment. The objective function included establishment, inventory, and transportation costs. They developed a bi-objective model for optimization of the mean and variance of costs and solved the model by genetic algorithm and simulated annealing.

In this paper, we assume two common objectives of minimizing total costs and maximizing purchasing value.

\subsection{Fuzzy multi-objective approaches}

There are some methods in the literature for aggregating fuzzy goals and constraints. Jiménez and Bilbao [28] proposed a method for multi-objective programming problem with fuzzy objectives to maximize $\sum \lambda_{i} f_{i}(x)$ subject to $\mu_{Z_{f}}(x) \geq \lambda$, with $\lambda$ representing the degree of satisfaction of DM with the achievement of goals. A unique optimal solution to the above problem is the efficient fuzzy solution to the original Multi Objective Problems (MOPs) [29]. There are some approaches such as the weighted additive approach [30,31], compromise approach [32], the method with achievement degrees [33], augmented max-min model $[34,35]$, and two-phase approach [36] in the literature, which ensure the existence of an efficient fuzzy solution. Moghaddam [23] applied fuzzy goal programming approach to the supplier selection and order allocation problem in reverse logistics systems. He utilized a Monte Carlo simulation integrated with fuzzy goal programming to determine the entire set of Pareto optimal solutions. By incorporating the linear membership functions for objectives and constraints in other constraints, he formulated a fuzzy goal programming model. Erginel and Gecer [37] investigated a fuzzy multi-objective linear programming model for the supplier selection problem. Weight, cost, and calibration time were handled as fuzzy numbers for modelling the imprecise data. A two-phase approach was used to obtain a Pareto optimal solution and solve the fuzzy multi-objective decision model. Çebi and Otay [25] studied the supplier selection and order allocation problem considering quantity discounts and lead time. They applied a two-stage fuzzy approach to solving the problem and used the augmented maxmin model, guaranteeing non-dominated solutions, to transform the fuzzy multi-objective model into a crisp single-objective one. To select suppliers, they utilized fuzzy MULTIMOORA at the first stage and fuzzy goal programming, to determine order amounts at the second stage. Govindan et al. [38] addressed the supplier selection problem with transportation decisions in an eco-efficient closed-loop supply chain network by utilizing weighted fuzzy mathematical programming approach to generating a properly efficient fuzzy solution. They first defined linear membership functions for each fuzzy goal as introduced by Zadeh [39] and then, applied a weighted max-min approach to searching for an optimal solution. Their aim was to make the levels of achievement of the goals and the weights of the goals as close to each other as possible.

As observed in the review of the literature, all fuzzy approaches transform a model into a singleobjective one. However, in this paper, we propose a method to solve a problem in its original multiobjective form.

\section{Problem formulation}

Assume that a supply chain includes a buyer and several suppliers. The buyer intends to evaluate suppliers and allocate orders to them, as shown in Figure 1. Thus, the buyer faces a supplier selection and 


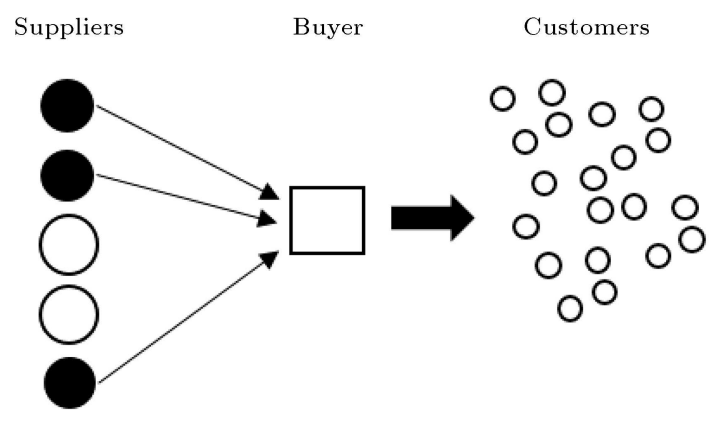

Figure 1. Schematic view of supply chain.

order allocation problem. The main objectives of the buyer are to minimize annual supply chain costs and maximize $A P V$ so that they do not face any shortages. The main assumptions of the problem are as follows:

- There is only one single product involved; the buyer can purchase their required amount from several suppliers;

- The annual demand is known and constant over time;

- Each supplier has a specific production capacity;

- Inventory shortage is not allowed for any supplier or buyer;

- The inventory cannot be transferred from period to period;

- The transportation cost from each supplier to the buyer depends on the distance and the number of required vehicles;

- The defective rate of each supplier is known;

- In each period, the $(i+1)$ th order by the supplier cannot be entered unless the whole $i$ th order is consumed;

- All suppliers use the price discount policy to encourage the buyer to place large orders. Suppose that there are $n$ suppliers in the supply chain. Each supplier $i$ uses the quantity discount policy, which includes $K_{i}$ price levels, and the $k$ th price level is determined by price $c_{i k}$ and order range $\left[u_{i, k-1}, u_{i k}\right)$. The logical assumption is that for $u_{i 1}<u_{i 2}<\ldots<$ $u_{i, K_{i}}$, we have $c_{i 1}>c_{i 2}>\ldots>c_{i, K_{i}}$.

The model studied in this research has some similarities with those of Kamali et al. [20] and Alaei and Khoshalhan [40]. They also considered the problem as a multi-objective programming model for maximizing $A P V$ and minimizing annual supply chain costs, defective items, and late deliveries. To solve the model, Kamali et al. [20] utilized PSO and SS; in addition to PSO and SS, Alaei and Khoshalhan [40] used the harmony search and the hybrid harmonycultural algorithm. However, while the above papers both dealt with the problem in deterministic environment, in this study, the problem is considered as a biobjective optimization model with fuzzy parameters for objectives and constraints. Therefore, a novel method is proposed for obtaining Pareto solutions to the fuzzy bi-objective problem. The method is an extension of the single-objective method proposed by Jiménez et al. [10]. We also utilize NSGA II to solve the problem.

\subsection{Notation}

The following parameters and decision variables are to be used in the model.

\section{Parameters}

$D \quad$ Annual demand rate of the buyer

$S_{i} \quad$ Fixed production setup cost to supplier

$$
i
$$

$A_{i} \quad$ Fixed ordering cost for supplier $i$

$h_{i} \quad$ Inventory holding cost to supplier $i$ per unit per unit time

$h_{b} \quad$ Inventory holding cost to buyer per unit per unit time

$T_{i} \quad$ Consumption time of an order quantity from supplier $i$

$T \quad$ Length of period for the buyer

$c_{i} \quad$ Variable cost per unit (fuzzy) to supplier $i$

$c_{i k} \quad$ Discounted unit price of interval $k$ offered by supplier $i$

$P_{i} \quad$ Production rate of supplier $i$

$u_{i k} \quad$ Upper bound of discount interval for supplier $i$

$r_{i} \quad$ Defective rate (fuzzy) for supplier $i$

$R \quad$ Maximum acceptable defective rate for all purchased quantities

$d i s_{i} \quad$ Distance between buyer and supplier $i$

cap Capacity of vehicles

C $\quad$ Fixed cost of transportation per distance unit (fuzzy)

$W_{i} \quad$ Importance rate of supplier $i$ in supplier evaluation methods (fuzzy)

\section{Decision variables}

$q_{i k} \quad$ Purchased quantity per period from supplier $i$ in discount interval $k$

$y_{i k} \quad$ Binary variable equal to 1 if $q_{i k}$ falls into discount interval $k$ of supplier $i, 0$ otherwise

$Q_{i} \quad$ Order quantity from supplier $i$ per period

$Q \quad$ Total order quantity per period from all suppliers

$Z_{i} \quad$ Integer variable denoting the number of required vehicles for transporting $Q_{i}$ 


\subsection{Model}

The mathematical modeling of the problem is shown as a mixed-integer nonlinear programming model as follows:

$$
\begin{aligned}
& \text { Min Cost }=\frac{D}{Q}\left[\sum_{i=1}^{n} \sum_{k=1}^{K_{i}}\left(\tilde{c}_{i}+c_{i k}\right) q_{i k}\right. \\
& +\sum_{i=1}^{n} \sum_{k=1}^{K_{i}}\left(A_{i}+S_{i}\right) y_{i k} \\
& \left.+\sum_{i=1}^{n} \frac{Q_{i}^{2}}{2}\left(\frac{h_{b}}{D}+\frac{h_{i}}{P_{i}}\right)+\sum_{i=1}^{n} \tilde{C} d i s_{i} Z_{i}\right] \\
& \operatorname{Max} \quad A P V=\frac{D}{Q} \sum_{i=1}^{n} \tilde{w}_{i} Q_{i}, \\
& Q_{i}=\sum_{k=1}^{K_{i}} q_{i k} \quad \forall i=1, \ldots, n, \\
& Q=\sum_{i=1}^{n} Q_{i} \\
& \frac{D}{Q} Q_{i} \leq P_{i} \quad \forall i=1, \ldots, n, \\
& \operatorname{cap}\left(Z_{i}-1\right) \leq Q_{i} \quad \forall i=1, \ldots, n, \\
& Q_{i} \leq \operatorname{cap}_{i} \quad \forall i=1, \ldots, n, \\
& \sum_{k=1}^{K_{i}} y_{i k} \leq 1 \quad \forall i=1, \ldots, n, \\
& u_{i, k-1} y_{i k} \leq q_{i k} \quad \forall i=1, \ldots n ; \quad \forall k=1, \ldots, K_{i}, \\
& q_{i k} \leq u_{i k} y_{i k} \quad \forall i=1, \ldots, n ; \quad \forall k=1, \ldots, K_{i}, \\
& \frac{1}{Q} \sum_{i=1}^{n} Q_{i} \tilde{r}_{i} \leq R \\
& y_{i k} \in\{0,1\} \quad \forall k=1: K_{i}, \quad i=1, \ldots, n, \\
& Z_{i} \in \text { Integer } \quad \forall i=1, \ldots, n, \\
& q_{i k} \geq 0 \quad \forall k=1: K_{i}, \quad i=1, \ldots, n, \\
& Q_{i} \geq 0 \quad \forall i=1, \ldots, n, \\
& Q \geq 0 \text {. }
\end{aligned}
$$

Eq. (1) minimizes the cost objective function, which consists of four parts. The first part involves variable and purchase costs; the second part includes ordering and setup costs; the third part is the inventory holding costs to the buyer and to suppliers; and the fourth part calculates the transportation costs. Eq. (2) maximizes the total $A P V$, which determines the weighted quantities of orders. These weights specify the importance of suppliers and can be the output of the multi-criteria decision-making methods [25]. This maximization relation leads to allocating more orders to more important suppliers. Eq. (3) states that the sum of purchases in the discount intervals of a supplier is equal to the amount of purchases from that supplier. Based on Eq. (4), the total purchase of the buyer in each cycle is equal to the sum of purchases from all suppliers. Capacity constraints of the suppliers are expressed by Eq. (5). The number of required vehicles to carry the products of each supplier is calculated using Eqs. (6) and (7). Eq. (8) ensures that the order to each supplier is only at one of its discount intervals. Through Eqs. (9) and (10), the order to each supplier falls into one of the discount intervals offered by the supplier. Eq. (11) ensures that the rate of defective products does not exceed a predetermined value. Finally, Relations (12)-(16) define the types of variables.

\section{Fuzzy multi-objective approach}

Various methods have been presented in the literature for fuzzy multi-objective optimization. However, we propose a novel method for the problem, which is based on satisfaction degree of the DM with fuzzy objectives and the level of fulfilment of the constraints. This method is an extension of the single-objective fuzzy programming proposed by Jimenez et al. [10] to the multi-objective environment. The method aims to identify the Pareto solutions which best match the desires of the DM. Fuzzy objective functions are replaced by the degree of satisfaction defined in the algorithm and fuzzy constraints are rewritten as a function of $\alpha$, which represents the level of fulfilment.

Step 1. According to Jiménez et al. [10], fuzzy constraints must be rewritten as a function of alpha. With these modifications, the problem space becomes wider for $\alpha=0$ and more limited for $\alpha=1$. For any constraint type " $\leq$," fuzzy coefficient $\tilde{a}=\left(a_{1}, a_{2}, a_{3}\right)$ and fuzzy right-hand side $\tilde{b}=\left(b_{1}, b_{2}, b_{3}\right)$ should be replaced by the following expressions, respectively:

$$
\begin{aligned}
& \tilde{a} \leftarrow(1-\alpha)\left(\frac{a_{1}+a_{2}}{2}\right)+\alpha\left(\frac{a_{2}+a_{3}}{2}\right), \\
& \tilde{b} \leftarrow \alpha\left(\frac{b_{1}+b_{2}}{2}\right)+(1-\alpha)\left(\frac{b_{2}+b_{3}}{2}\right) .
\end{aligned}
$$

Step 2. The DM is asked to specify the interval $(G, \bar{G})$ for each objective. For a minimization objective, if $z \leq \underline{G}$, they will find it totally satisfactory; but if $z \geq \bar{G}$, their degree of satisfaction will be null. 
Accordingly, the goal is expressed by means of a fuzzy set $\tilde{G}$ whose membership function is as follows:

$$
\mu_{\tilde{G}}(z)= \begin{cases}1 & \text { if } z \leq \underline{G} \\ \lambda \epsilon[0,1] & \text { decreasing on } \quad \underline{G} \leq z \leq \bar{G} \\ 0 & \text { if } z \geq \bar{G}\end{cases}
$$

Similarly, we define $\tilde{G}$ for maximization objectives as follows.

$$
\mu_{\tilde{G}}(z)= \begin{cases}1 & \text { if } z \leq \underline{G} \\ \lambda \epsilon[0,1] & \text { increasing on } \quad \underline{G} \leq z \leq \bar{G} \\ 0 & \text { if } z \geq \bar{G}\end{cases}
$$

The DM aims to gain the maximum degree of satisfaction. However, in order to get a better objective value, a lower level of fulfilment of constraints is considered. Given these circumstances, the DM might want a lower satisfaction degree of objectives in exchange for a better level of fulfilment of constraints [10].

Step 3. For each objective, we need to compute the satisfaction degree of the fuzzy goal $\tilde{G}$ by each $\alpha$ acceptable Pareto solution, that is, the membership degree of each fuzzy number $\tilde{z}^{0}\left(\alpha_{k}\right)$ in the fuzzy set $\tilde{G}$. There are several methods for this purpose (e.g., [41]. We will apply the index proposed by Yager [42] and used by Jiménez et al. [10] as follows:

$$
K_{\tilde{G}}\left(z^{0}(\alpha)\right)=\frac{\int_{-\infty}^{+\infty} \mu_{\tilde{z}^{0}(\alpha)}(z) \times \mu_{\tilde{G}}(z) d z}{\int_{-\infty}^{+\infty} \mu_{\tilde{z}^{0}(\alpha)}(z) d z},
$$

where the denominator is the area under $\mu_{\tilde{z}^{0}(\alpha)}$ and, in the numerator, the possibility of occurrence $\mu_{\tilde{z}^{0}(\alpha)}(z)$ for each crisp value $z$ is weighted by its satisfaction degree $\mu_{\tilde{G}}(z)$ for goal $\tilde{G}$ (see Figure 2).

Step 4. Here, in order to achieve a balance between satisfaction degree and constraints fulfilment level, we use the condition similar to that considered by Jiménez et al. [10] as follows:

$$
x^{*}\left(\alpha_{k}\right): \alpha_{k}=\operatorname{argmax}_{\alpha_{k}}\left\{\alpha_{k} \times K_{\tilde{G}}\left(\tilde{z}^{0}\left(\alpha_{k}\right)\right)\right\} \text {. }
$$

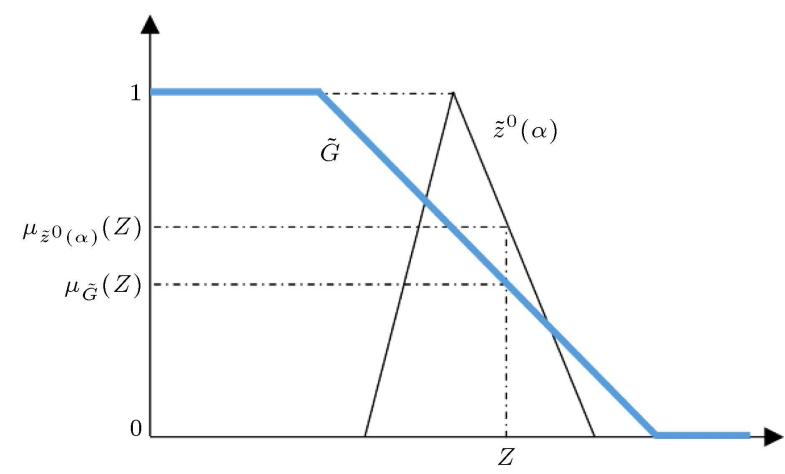

Figure 2. Occurrence possibility of a crisp objective value, $z$, and its goal satisfaction degree.
The greater the value of $\alpha$, the more limited the problem space and the lower the satisfaction degree and vice versa. By using the above equation, a tradeoff between $\alpha$ and satisfaction degree can be achieved.

Step 5. The solutions for each fuzzy objective function can be compared and the DM can identify the appropriate or inappropriate solutions among Pareto solutions through the following two definitions:

Definition 1: For any pair of fuzzy numbers $\tilde{a}$ and $\tilde{b}$, the degree in which $\tilde{a}$ is greater than $\tilde{b}$ is calculated as follows [10]:

$$
\begin{aligned}
& \mu_{M}(\tilde{a}, \tilde{b})= \\
& \begin{cases}0 & \text { if } E_{2}^{a}-E_{1}^{b}<0 \\
\frac{E_{2}^{a}-E_{1}^{b}}{\left(E_{2}^{a}-E_{1}^{a}\right)+\left(E_{2}^{b}-E_{1}^{b}\right)} & \text { if }\left[E_{1}^{a}-E_{2}^{b}, E_{2}^{a}-E_{1}^{b}\right]<0 \\
1 & \text { if } E_{1}^{a}-E_{2}^{b}>0\end{cases}
\end{aligned}
$$

where $\left[E_{1}^{a}, E_{2}^{a}\right]$ and $\left[E_{1}^{b}, E_{2}^{b}\right]$ are the expected intervals of $\tilde{a}$ and $\tilde{b}$. We say that $\tilde{a}$ and $\tilde{b}$ are indifferent if $\mu_{M}(\tilde{a}, \tilde{b})=0.5$.

Definition 2: For a triangular fuzzy number $\tilde{a}=$ $\left(a_{1}, a_{2}, a_{3}\right)$, the expected interval is easily calculated as follows [43]:

$$
E I(\tilde{a})=\left[E_{1}^{a}, E_{2}^{a}\right]=\left[\frac{a_{1}+a_{2}}{2}, \frac{a_{2}+a_{3}}{2}\right] .
$$

\subsection{The case of trapezoidal fuzzy numbers}

The proposed method can also be applied to trapezoidal fuzzy numbers. In Step 1, for any fuzzy coefficient $\tilde{a}=\left(a_{1}, a_{2}, a_{3}, a_{4}\right)$ and fuzzy right-hand side $\tilde{b}=\left(b_{1}, b_{2}, b_{3}, b_{4}\right)$, Relations (17) and (18) can be rewritten as [10]:

$$
\begin{aligned}
& \tilde{a} \leftarrow(1-\alpha)\left(\frac{a_{1}+a_{2}}{2}\right)+\alpha\left(\frac{a_{3}+a_{4}}{2}\right), \\
& \tilde{b} \leftarrow \alpha\left(\frac{b_{1}+b_{2}}{2}\right)+(1-\alpha)\left(\frac{b_{3}+b_{4}}{2}\right) .
\end{aligned}
$$

In Definition 2 of Step 5, for a fuzzy number $\tilde{a}=$ $\left(a_{1}, a_{2}, a_{3}, a_{4}\right)$, the expected interval will be [43]:

$$
E I(\tilde{a})=\left[E_{1}^{a}, E_{2}^{a}\right]=\left[\frac{a_{1}+a_{2}}{2}, \frac{a_{3}+a_{4}}{2}\right] .
$$

The other steps remain unchanged.

\section{Solution procedure}

The defined problem in the previous section is nonlinear. The reason is that the variable $Q$ is the denominator of Eqs. (1), (2), (5), and (11). Certainly, Eq. (5) can be converted to a linear form, $D Q_{i} \leq P_{i} Q$. The linear form of Eq. (11) can also be rewritten as $\sum_{i=1}^{n} Q_{i} \tilde{r}_{i} \leq R Q$. 
However, Relations (1) and (2) are totally nonlinear. Considering that nonlinear problems cannot be solved by exact methods, NSGA II is designed for solving the problem in hand. Firstly, the fuzzy objective functions and the fuzzy constraints must be modified in accordance with the proposed method.

\subsection{Modification of fuzzy objectives and fuzzy constraints}

Given the first step of the proposed method, assuming that $\widetilde{r_{i}}=\left(r_{i}^{1}, r_{i}^{2}, r_{i}^{3}\right)$, Eq. (11) can be rewritten as follows:

$$
\sum_{i=1}^{n} Q_{i}\left[(1-\alpha)\left(\frac{r_{i}^{1}+r_{i}^{2}}{2}\right)+\alpha\left(\frac{r_{i}^{2}+r_{i}^{3}}{2}\right)\right] \leq \underset{(28}{Q R .}
$$

It is also assumed that the fuzzy values of the objective functions in a solution are denoted by $\widetilde{\text { Cost }}=$ $\left(\rho_{1}, \rho_{2}, \rho_{3}\right)$ and $\widetilde{A P V}=\left(\delta_{1}, \delta_{2}, \delta_{3}\right)$. The interval $(\underline{G}, \bar{G})$ of each objective can be determined by the DM. Here, we assume that the ideal value of each objective is determined by optimizing the function and ignoring the other one. Also, the non-ideal value is determined by the DM. In optimizing each objective, the problem is solved for the smallest possible amount of $\alpha$, which ensures that the problem space is in the broadest state. Then, the degree of satisfaction of objectives can be calculated as follows:

$$
\begin{aligned}
& K_{\tilde{G}}\left(\operatorname{Cost}^{0}(\alpha)\right)= \\
& \frac{\int_{\rho_{1}}^{\rho_{2}}\left(\frac{z-\rho_{1}}{\rho_{2}-\rho_{1}}\right) \times\left(\frac{z-\bar{G}}{\underline{G}-\bar{G}}\right) d z+\int_{\rho_{2}}^{\rho_{3}}\left(\frac{z-\rho_{3}}{\rho_{2}-\rho_{3}}\right) \times\left(\frac{z-\bar{G}}{\underline{G}-\bar{G}}\right) d z}{\int_{\rho_{1}}^{\rho_{2}}\left(\frac{z-\rho_{1}}{\rho_{2}-\rho_{1}}\right) d z+\int_{\rho_{2}}^{\rho_{3}}\left(\frac{z-\rho_{3}}{\rho_{2}-\rho_{3}}\right) d z}, \\
& \left.\left.\frac{K_{\tilde{G}}\left(A P V^{0}(\alpha)\right)=}{\int_{\delta_{1}}^{\delta_{2}}\left(\frac{z-\delta_{1}}{\delta_{2}-\delta_{1}}\right) \times\left(\frac{z-\underline{G}}{\bar{G}-\underline{G}}\right) d z+\int_{\delta_{2}}^{\delta_{3}}\left(\frac{z-\delta_{3}}{\delta_{2}-\delta_{3}}\right) \times\left(\frac{z-\underline{G}}{\bar{G}-\underline{G}}\right) d z} \delta_{\delta_{3}}^{\delta_{2}-\delta_{1}}\right) d z+\frac{z-\delta_{3}}{\delta_{2}-\delta_{3}}\right) d z
\end{aligned}
$$

It should be noted that the objective functions in Eqs. (1) and (2) are minimization and maximization, respectively. Thus, the membership function of $\tilde{G}$ is descending for the first objective and ascending for the second one. The integrals of the above relations can be easily calculated.

\subsection{Solution representation}

Each chromosome or answer vector can be expressed as $Q:\left[Q_{1}, Q_{2}, \ldots, Q_{n}\right]$; the sum of the vector is equal to $Q . Q_{i}$ represents the order quantity assigned to supplier $i$ and it is equal to $Q_{i}:\left[q_{i 1}, q_{i 2}, \ldots, q_{i, K_{i}}\right]$. The procedure for generating initial solutions is shown in Figure 3.
For each suppliesr $i$ from 1 to $n$, do:

Generate a random number between $(0,1)$ and name it Rand If $\operatorname{Rand}<0.5$

Assign a random number between $\left[0, u_{i, K_{i}}\right)$ to $Q_{i}$ Otherwise set $Q_{i}=0$

Figure 3. Procedure of the initial solution generation.

\subsection{Repair algorithm}

Due to the existence of constraints in the problem, it is possible to face infeasible solutions in the initial solution generation algorithm and repetition of the main algorithm. These infeasible solutions must be controlled using the constraints handling methods. In this section, we propose a repair algorithm, which transforms infeasible solutions to feasible ones. Eqs. (3) and (4) are established by solution representation. By calculating the number of vehicles, Eqs. (6) and (7) are enforced. Eqs. (8)-(10) are also established according to the order quantity from each supplier, which should fall into one of the discount intervals. The only constraints that may lead to infeasible solutions are Eqs. (5) and (25). In the following, we propose a repair algorithm for them:

- Repair Algorithm 1: In an infeasible solution, according to Eq. (5), for each supplier $i$, we define $a_{i}=P_{i}-D Q_{i} / Q$. If $a_{i}>0$, then the supplier still has some capacity for order assignment. On the other hand, if $a_{i}<0$, the amount of annual order assigned to the supplier exceeds its annual production capacity. Therefore, we define two sets of $S^{+}=\left\{i: a_{i} \geq 0\right\}$ and $S^{-}=\left\{i: a_{i} \leq 0\right\}$. The set $S^{+}$represents the suppliers with an additional capacity for assignment and the set $S^{-}$represents the suppliers whose capacity constraints are violated. The following changes ensure the feasibility of a solution if we have $\sum_{i \in S^{+}} a_{i} \geq \sum_{i \in S^{-}} a_{i}$; otherwise, the solution is rejected:

$$
\begin{aligned}
& Q_{i}=\frac{Q}{D} P_{i} \quad \forall i \in S^{-}, \\
& Q_{i}=Q_{i}+\left(\frac{a_{i}}{\sum_{i \in S^{+}} a_{i}}\right) \sum_{i \in S^{-}} a_{i} \quad \forall i \in S^{+} .
\end{aligned}
$$

According to Eq. (31), the annual order quantity from suppliers with violated capacity constraint is set equal to their annual production rate. Eq. (32) shares the additional order quantity $\left(\sum_{i \in S^{-}} a_{i}\right)$ among other unviolated suppliers in proportion to their available capacities.

- Repair Algorithm 2: For Eq. (28), we define:

$$
A=\sum_{i=1} Q_{i} r_{i^{\prime}}-Q R
$$

in which: 


$$
r_{i^{\prime}}=\left[(1-\alpha)\left(r_{i}^{1}+r_{i}^{2}\right)+\alpha\left(r_{i}^{2}+r_{i}^{3}\right)\right] / 2 .
$$

Also, due to changes in Repair Algorithm 1, $a_{i}$ is re-calculated for all suppliers and the sets $S^{+}$and $S^{-}$are formed. If we have $A>0$, the constraint is violated. Thus, two suppliers $i$ and $j$ are randomly chosen, provided that we have $r_{i}^{\prime}>r_{j}^{\prime}$ and $j \in S^{+}$. Then, modifications $Q_{j}=Q_{j}+\Delta^{\prime}$ and $Q_{i}=Q_{i}-$ $\Delta^{\prime}$ must be applied. In this case, it is guaranteed that the total order quantity $(Q)$ is not changed. Reducing the order quantity from supplier $i$ does not lead to any violation. However, increasing it may lead to exceeding the production capacity constraint. Therefore, consider the following changes:

$$
\begin{aligned}
\Delta & =\frac{A}{r_{i}^{\prime}-r_{j}^{\prime}}, \\
\Delta^{\prime} & =\min \left\{\Delta, a_{j}, Q_{i}\right\} .
\end{aligned}
$$

Eq. (33) determines the amount which must be reduced from the order quantity from supplier $i$ and added to the order quantity from supplier $j$. This change should be considered in accordance with Repair Algorithm 1. Therefore, according to Eq. (34), the minimum difference between the amount determined in Eq. (33) and the remaining capacity of supplier $j, a_{j}$, must be selected. Also, to prevent negative values for $Q_{i}$, the minimum difference between $Q_{i}$ and the determined value should be chosen. It should be noted that the procedure is repeated until the value of $A$ is positive.

If repair is not performed within the predefined replications, the solution modifications of Repair Algorithm 2 are ignored and Repair Algorithms 1 and 2 are repeated again.

\subsection{Non-dominated Sorting Genetic Algorithm (NSGA II)}

NSGA II was presented by Deb et al. [44] as one of the best algorithms for obtaining Pareto frontiers. In this algorithm, the population $P_{1}$ is generated with respect to population size $N_{p}$. At repetition $t$, after selecting parent chromosomes from population $P_{t}$, the offspring (population $O_{t}$ ) are generated according to the crossover rate $p_{c}$ and the mutation rate $p_{m}$. Then, $P_{t}$ is merged with $O_{t}$ and chromosomes are sorted into non-dominated frontiers based on their rank and crowding distance. $N_{p}$ best solutions form population $P_{t+1}$. The algorithm continues until the best Pareto solutions are obtained in accordance with the stop condition. For more information, see Deb et al. [44] and Deb [45].

A graphical view of the algorithm is shown in Figure 4. After non-dominated sorting, the solutions are sorted into $F_{i}$ frontiers, where $F_{1}$ is the best Pareto frontier. The chromosomes within each frontier are also sorted according to the crowding distance.

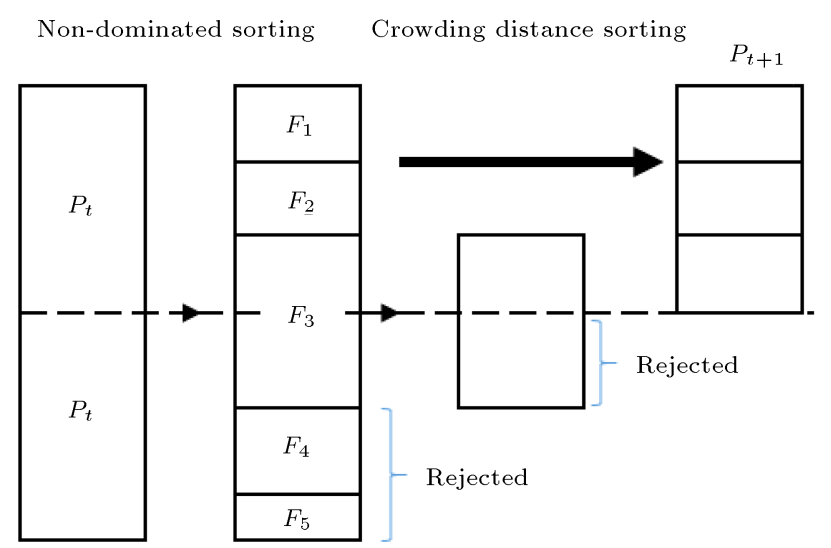

Figure 4. Graphical view of NSGA II.

After merging parents and offspring populations, the best frontiers are transferred to the new population. As shown in Figure 4 , the frontiers $F_{1}$ and $F_{2}$ and the chromosomes with higher crowding distances on frontier $F_{3}$ are transferred to the new population and the other frontiers are eliminated.

In the proposed algorithm, we utilize a uniform crossover. Assume that chromosomes $m$ and $n$ are selected. After choosing a random number $\tau$ in the interval $(0,1)$, the order quantities for each supplier $i$ in offspring 1 and 2 are determined by $Q_{i}^{1}=\tau Q_{i}^{m}+$ $(1-\tau) Q_{i}^{n}$ and $Q_{i}^{2}=(1-\tau) Q_{i}^{m}+\tau Q_{i}^{n}$, respectively. In addition, if a mutation is applied to the chromosome, one supplier is randomly selected and its order quantity is exactly determined by the initial solution generation procedure.

\section{Computational results}

An example is presented in this section. All the required data, except for fuzzy data and the data related to vehicles, are taken from Kamali et al. [20]. A buyer with an annual demand of 100,000 units plans to purchase the required amount from 4 suppliers. The inventory holding cost to the buyer is $\$ 2.6$ and the capacity of each vehicle is 5,000 units. The information on the suppliers is shown in Table 1. The fixed cost of each vehicle per unit of distance is assumed as a fuzzy number $C=(400,530,640)$. Other fuzzy parameters

Table 1. Information on the suppliers in the example.

\begin{tabular}{ccccc}
\hline Parameter & \multicolumn{4}{c}{ Supplier } \\
\cline { 2 - 5 } & $\mathbf{1}$ & $\mathbf{2}$ & $\mathbf{3}$ & $\mathbf{4}$ \\
\hline$S$ & 43 & 39 & 42 & 30 \\
$P$ & 35108 & 29898 & 35785 & 68777 \\
$A$ & 40 & 19 & 25 & 39 \\
$h$ & 2.29 & 1.96 & 2.74 & 0.54 \\
dis & 25 & 20 & 15 & 17 \\
\hline
\end{tabular}


Table 2. Fuzzy parameters in the example.

\begin{tabular}{cccc}
\hline Supplier & $\tilde{\boldsymbol{c}}$ & $\tilde{\boldsymbol{w}}$ & $\tilde{\boldsymbol{r}}$ \\
\hline 1 & $(3,4.04,4.9)$ & $(0.4,0.44,0.48)$ & $(0.0307,0.0344,0.0389)$ \\
2 & $(6,6.48,7.12)$ & $(0.55,0.64,0.67)$ & $(0.0498,0.0551,0.0674)$ \\
3 & $(7,7.17,7.8)$ & $(0.71,0.72,0.78)$ & $(0.0116,0.0121,0.0149)$ \\
4 & $(5,5.87,6.23)$ & $(0.55,0.57,0.62)$ & $(0.0205,0.0215,0.0265)$ \\
\hline
\end{tabular}

Table 3. Quantity discounts offered by suppliers.

\begin{tabular}{|c|c|c|}
\hline Supplier & Unit price & Order interval \\
\hline \multirow{7}{*}{1} & 9 & $(0,5000)$ \\
\hline & 8.9 & {$[5000,10000)$} \\
\hline & 8.8 & {$[10000,15000)$} \\
\hline & 8.7 & {$[15000,20000)$} \\
\hline & 8.6 & {$[20000,25000)$} \\
\hline & 8.5 & {$[25000,30000)$} \\
\hline & 8.4 & {$[30000,35108)$} \\
\hline \multirow{6}{*}{2} & 9.1 & {$[0,2000)$} \\
\hline & 9 & {$[2000,4000)$} \\
\hline & 8.9 & {$[4000,6000)$} \\
\hline & 8.8 & {$[6000,8000)$} \\
\hline & 8.7 & {$[8000,10000)$} \\
\hline & 8.6 & {$[10000,20000)$} \\
\hline \multirow{8}{*}{3} & 8.7 & {$[0,3000)$} \\
\hline & 8.6 & {$[3000,6000)$} \\
\hline & 8.5 & {$[6000,9000)$} \\
\hline & 8.4 & {$[9000,12000)$} \\
\hline & 8.3 & {$[12000,15000)$} \\
\hline & 8.2 & {$[15000,18000)$} \\
\hline & 8.1 & {$[18000,21000)$} \\
\hline & 8 & {$[21000,30000)$} \\
\hline \multirow{5}{*}{4} & 10.5 & {$[0,4000)$} \\
\hline & 10.4 & {$[4000,8000)$} \\
\hline & 10.3 & {$[8000,12000)$} \\
\hline & 10.2 & {$[12000,16000)$} \\
\hline & 10.1 & {$[16000,68777)$} \\
\hline
\end{tabular}

are also given in Table 2. In addition, the discount price offered by suppliers is shown in Table 3 .

Let us denote fuzzy objectives by $\widetilde{\text { Cost }}=$ $\left(\rho_{1}, \rho_{2}, \rho_{3}\right)$ and $\overparen{A P V}=\left(\delta_{1}, \delta_{2}, \delta_{3}\right)$. According to the proposed method, we need to determine the interval $(\underline{G}, \bar{G})$ for each objective. For this purpose, two singleobjective optimization models are solved for $\alpha=0$. By minimizing $\rho_{1}$, the fuzzy optimal solution is obtained as $\widehat{\text { Cost }}=(\underline{1584200}, 1698800,1819600)$. Also, by maximizing $\delta_{3}$, the fuzzy optimal solution is $\widehat{A P V}=$ $(67000,70000, \underline{75000})$. Given the optimal solutions to
Table 4. Pareto solutions to the problem with respect to different values of $\alpha$.

\begin{tabular}{cccccc}
\hline$\#$ & $\boldsymbol{\alpha}$ & $\boldsymbol{K}_{\mathbf{1}}$ & $\boldsymbol{K}_{\mathbf{2}}$ & $\boldsymbol{\alpha} \boldsymbol{K}_{\mathbf{1}}$ & $\boldsymbol{\alpha} \boldsymbol{K}_{\mathbf{2}}$ \\
\hline 1 & 0.1 & 0.6358 & 0.7924 & 0.0635 & 0.0792 \\
2 & 0.1 & 0.7909 & 0.1831 & 0.0790 & 0.0183 \\
3 & 0.2 & 0.6355 & 0.797 & 0.1271 & 0.1594 \\
4 & 0.2 & 0.7861 & 0.2038 & 0.1572 & 0.0407 \\
5 & 0.3 & 0.5214 & 0.8016 & 0.1564 & 0.2404 \\
6 & 0.3 & 0.776 & 0.223 & 0.2328 & 0.0669 \\
7 & 0.4 & 0.6815 & 0.806 & 0.2726 & 0.3224 \\
8 & 0.4 & 0.7802 & 0.258 & 0.3120 & 0.1032 \\
9 & 0.5 & 0.4988 & 0.8102 & 0.2494 & 0.4051 \\
10 & 0.5 & 0.7865 & 0.2828 & 0.3932 & 0.1414 \\
11 & 0.6 & 0.6538 & 0.8143 & 0.3922 & 0.4885 \\
12 & 0.6 & 0.7822 & 0.3043 & 0.4693 & 0.1825 \\
13 & 0.7 & 0.6242 & 0.8183 & 0.4369 & 0.5728 \\
14 & 0.7 & 0.7806 & 0.3252 & 0.5464 & 0.2276 \\
15 & 0.8 & 0.6678 & 0.8221 & 0.5342 & 0.6576 \\
16 & 0.8 & 0.7561 & 0.3218 & 0.6048 & 0.2574 \\
17 & 0.9 & 0.6785 & 0.8259 & 0.6106 & 0.7433 \\
18 & 0.9 & 0.7816 & 0.3722 & 0.7034 & 0.3349 \\
19 & 1 & 0.5605 & 0.8295 & 0.5605 & 0.8295 \\
20 & 1 & 0.7698 & 0.3253 & 0.7698 & 0.3253 \\
\hline
\end{tabular}

the two optimization models, it is determined that $\underline{G}^{\text {Cost }}=1584200$ and $\bar{G}^{A P V}=75000$. The DM also sets the non-ideal values for both objective functions as $\bar{G}^{\text {Cost }}=2300000$ and $\underline{G}^{A P V}=55000$. Thus, the interval $(\underline{G}, \bar{G})$ for cost and $A P V$ will be (1584200, $2300000)$ and $(55000,75000)$, respectively.

Table 4 shows the Pareto solutions obtained by solving the problem for different values of $\alpha$. The problem has been solved for $\alpha=0.1,0.2, \ldots, 1$ and the satisfaction level of the DM has been reported. Also, normalized satisfaction levels are shown in the fifth and sixth columns.

Regarding the normalized satisfaction levels, if the dominated solutions are eliminated, solutions 1720 are identified as the final Pareto solutions. Figure 5 shows the remaining Pareto solutions.

Table 5 shows the order quantities assigned to suppliers in Pareto solutions. Also, the fuzzy membership functions for cost and $A P V$ objectives are shown 
Table 5. Order quantities in Pareto solutions.

\begin{tabular}{ccccc}
\hline & \multicolumn{4}{c}{ Supplier } \\
\cline { 2 - 5 }$\#$ & $\mathbf{1}$ & $\mathbf{2}$ & $\mathbf{3}$ & $\mathbf{4}$ \\
\hline 17 & 9986 & 0 & 16411 & 0 \\
18 & 0 & 3323 & 14717 & 0 \\
19 & 9950 & 0 & 18098 & 3860 \\
20 & 0 & 5924 & 27355 & 0 \\
\hline
\end{tabular}

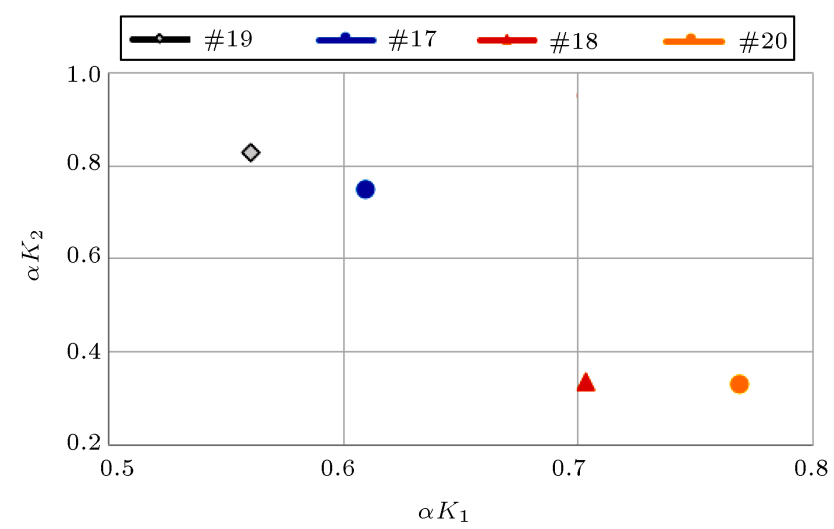

Figure 5. Final Pareto solutions with respect to the normalized satisfaction levels.

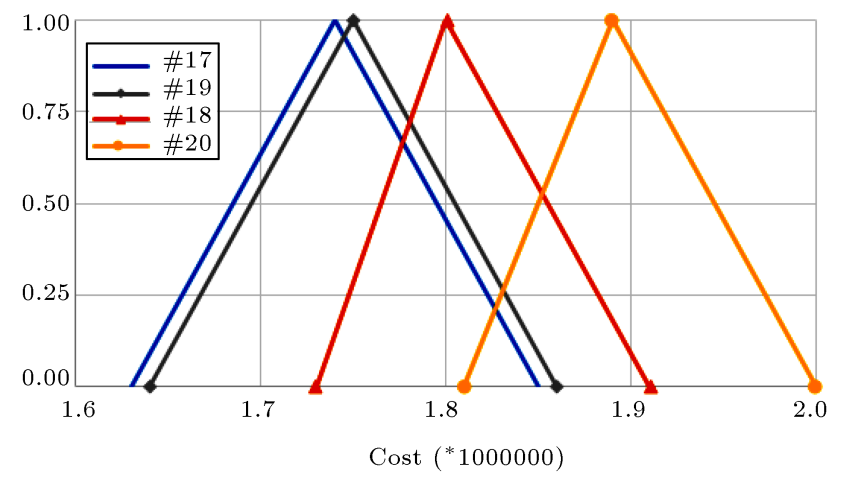

Figure 6. Fuzzy membership function of cost objective in Pareto solutions.

in Figures 6 and 7, respectively. According to them, the DM can choose the appropriate solution.

Given the fuzzy values of the cost in Figure 6, we can compare the solutions. According to the calculations of fuzzy cost values: (a) The degrees in which solution \#20 is bigger than solutions \#17, \#18, and \#19 are 1, 0.97, and 1, respectively; (b) The degrees in which solution \#18 is bigger than solutions \#17 and \#19 are 0.85 and 0.8 , respectively; and (c) The degree in which solution \#19 is bigger than solution \#17 is 0.55 . Given the minimization of the cost objective function, solution \#20 is the worst among all the solutions.

Similarly, we can compare the solutions given the fuzzy values of $A P V$ objective in Figure 7: (a) There is no difference between solutions \#17 and \#19; (b)

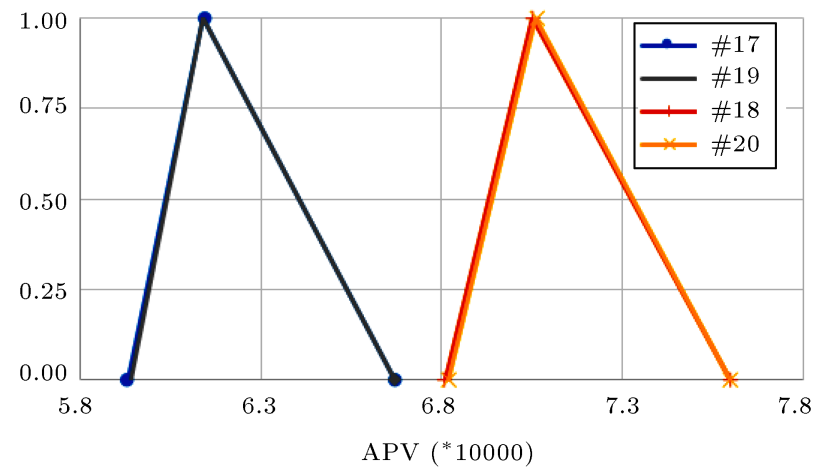

Figure 7. Fuzzy membership function of $A P V$ objective in Pareto solutions.

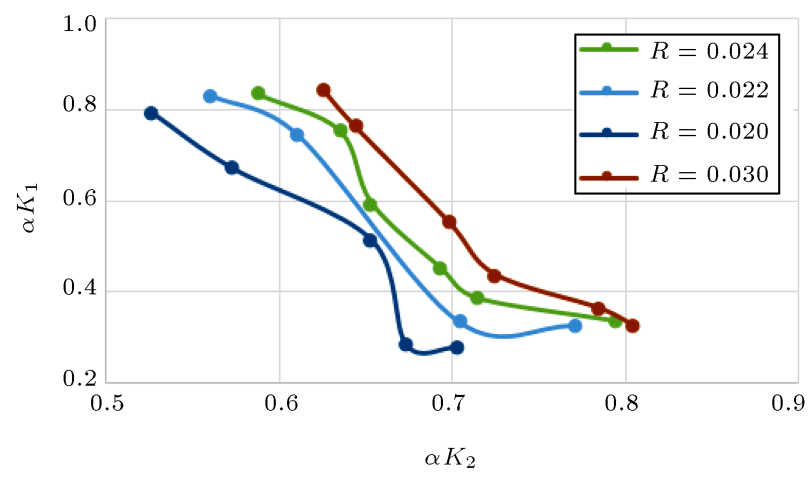

Figure 8. Pareto frontiers with regard to different values of $R$.

The degree in which both solutions \#18 and \#20 are bigger than either solution \#17 or \#19 is equal to 1; and (c) There is no difference between solutions \#18 and \#20. For indifferent solutions in $A P V$, one can choose a solution that is more cost-effective.

According to the analysis, there is no difference between solutions \#18 and \#20 in APV and the degree in which solution \#20 is bigger than \#18 is 0.97 . Therefore, solution \#18 is preferred to solution \#20. Similarly, there is no difference between solutions \#17 and \#19 in $A P V$ and the degree in which solution \#19 is bigger than \#17 is 0.55 . Therefore, solution \#17 is preferred to solution \#19.

In the example above, the maximum acceptable defective rate $(R)$ is assumed to be 0.022 . In the following, the problem is also solved for $R=0.02,0.024$, and 0.030 and the same procedure is followed to identify the final Pareto solutions. Figure 8 shows the results. In the maximization of satisfaction levels, the farther the Pareto frontier from the origin, the better the quality of its solutions will be. It is expected that by increasing $R$, the solution space gets wider and better results are obtained. Figure 8 also confirms this.

\section{Conclusion}

In the current competitive environment, supplier se- 
lection is one of the major processes in supply chain management of any organization. On the other hand, allocating orders to the selected suppliers allows for some economies of scale through the right choice of the quantities to order from each supplier. In this paper, the supplier selection and order allocation problem was studied in a single-buyer-multi-supplier supply chain. Suppliers offered quantity discounts as an incentive to motivate buyers to increase the amount of their ordered quantities. Also, transportation cost, fuzzytype uncertainty, and some practical constraints were taken into account in the problem. The problem was formulated as a bi-objective model to minimize annual supply chain costs and maximize the Annual Purchasing Value $(A P V)$. We proposed a novel fuzzy multi-objective programming method based on the degree of satisfaction of the Decision Maker (DM) and the fulfillment level of fuzzy constraints. After solving the model and determining Pareto solutions, the fuzzy results were compared with an index and the DM could identify the appropriate or inappropriate solutions. We utilized Non-dominated Sorting Genetic Algorithm (NSGA II) to solve the model and the results were presented using numerical examples. The interested researchers can also investigate demand uncertainty, the effect of different potential suppliers in each period, and other quantity discount schemes such as incremental quantity discounts and business volume quantity discounts in the problem. Also, other algorithms such as Multi-Objective Particle Swarm Optimization (MOPSO) and Multi-Objective Simulated Annealing (MOSA) can be applied to the problem in order to investigate the efficiency of the proposed NSGA II.

\section{References}

1. Hamdan, S. and Cheaitou, A. "Supplier selection and order allocation with green criteria: An MCDM and multi-objective optimization approach", Comput. Oper. Res., 81, pp. 282-304 (2017).

2. Govindan, K., Rajendran, S., Sarkis, J., et al. "Multi criteria decision making approaches for green supplier evaluation and selection: a literature review", $J$. Clean. Prod., 98, pp. 66-83 (2015).

3. Guo, C. and Li, X. "A multi-echelon inventory system with supplier selection and order allocation under stochastic demand", Int. J. Prod. Econ., 151, pp. 3747 (2014).

4. Kannan, D., Khodaverdi, R., Olfat, L., et al. "Integrated fuzzy multi criteria decision making method and multi-objective programming approach for supplier selection and order allocation in a green supply chain", J. Clean. Prod., 47, pp. 355-367 (2013).

5. Pazhani, S., Ventura, J.A., and Mendoza, A. "A serial inventory system with supplier selection and order quantity allocation considering transportation costs", Appl. Math. Model., 40(1), pp. 612-634 (2016).
6. Ballou, R.H., Business Logistics Management. Upper Saddle River, NY Prentice Hall (1992).

7. Mansini, R., Savelsbergh, M.W.P., and Tocchella, B. "The supplier selection problem with quantity discounts and truckload shipping", Omega, 40(4), pp. 445-455 (2012).

8. Taleizadeh, A.A., Stojkovska, I., and Pentico, D.W. "An economic order quantity model with partial backordering and incremental discount", Comput. Indust. Eng., 82, pp. 21-32 (2015).

9. Ayhan, M.B. and Kilic, H.S. "A two stage approach for supplier selection problem in multi-item/multisupplier environment with quantity discounts", Comput. Indust. Eng., 85, pp. 1-12 (2015).

10. Jiménez, M., Arenas, M., Bilbao, A., et al. "Linear programming with fuzzy parameters: an interactive method resolution", Eur. J. Oper. Res., 177(3), pp. 1599-1609 (2007).

11. Dahel, N.-E. "Vendor selection and order quantity allocation in volume discount environments", Suppl. Chain Manag.: Int. J., 8(4), pp. 335-342 (2003).

12. Demirtas, E.A. and Üstün, Ö. "An integrated multiobjective decision making process for supplier selection and order allocation", Omega, 36, pp. 76-90 (2008).

13. Xia, W. and Wu, Z. "Supplier selection with multiple criteria in volume discount environments", Omega, 35(5), pp. 494-504 (2007).

14. Burke, G.J., Carrillo, J., and Vakharia, A.J. "Heuristics for sourcing from multiple suppliers with alternative quantity discounts", Eur. J. Oper. Res., 186(1), pp. 317-329 (2008).

15. Kokangul, A. and Susuz, Z. "Integrated analytical hierarch process and mathematical programming to supplier selection problem with quantity discount", Appl. Math. Model., 33(3), pp. 1417-1429 (2009).

16. Amid, A., Ghodsypour, S.H., and O'Brien, C. "A weighted additive fuzzy multiobjective model for the supplier selection problem under price breaks in a supply chain", Int. J. Prod. Econ., 121(2), pp. 323332 (2009).

17. Wang, T.-Y. and Yang, Y.-H. "A fuzzy model for supplier selection in quantity discount environments", Expert Syst. Appl., 36(10), pp. 12179-12187 (2009).

18. Ebrahim, R.M., Razmi, J., and Haleh, H. "Scatter search algorithm for supplier selection and order lot sizing under multiple price discount environment", Adv. Eng. Soft., 40(9), pp. 766-776 (2009).

19. Razmi, J. and Maghool, E. "Multi-item supplier selection and lot-sizing planning under multiple price discounts using augmented e-constraint and Tchebycheff method", Int. J. Adv. Manuf. Technol., 49(1-4), pp. 379-392 (2010).

20. Kamali, A., Fatemi-Ghomi, S., and Jolai, F. "A multiobjective quantity discount and joint optimization model for coordination of a single-buyer multi-vendor supply chain", Comput. Math. Appl., 62(8), pp. 32513269 (2011). 
21. Zhang, J.L. and Zhang, M.Y. "Supplier selection and purchase problem with fixed cost and constrained order quantities under stochastic demand", Int. J. Prod. Econ., 129(1), pp. 1-7 (2011).

22. Lee, A.H.I., Kang, H.-Y., Lai, C.-M., et al. "An integrated model for lot sizing with supplier selection and quantity discounts", Appl. Math. Model., 37(7), pp. 4733-4746 (2013).

23. Moghaddam, K.S. "Fuzzy multi-objective model for supplier selection and order allocation in reverse logistics systems under supply and demand uncertainty", Expert Syst. Appl., 42, pp. 6237-6254 (2015).

24. Bohner, C. and Minner, S. "Supplier selection under failure risk, quantity and business volume discounts", Comput. Indust. Eng., 104, pp. 145-155 (2017).

25. Çebi, F. and Otay, İ. "A two-stage fuzzy approach for supplier evaluation and order allocation problem with quantity discounts and lead time", Inf. Sci., 339, pp. 143-157 (2016).

26. Hamdan, S. and Cheaitou, A. "Dynamic green supplier selection and order allocation with quantity discounts and varying supplier availability", Comput. Indust. Eng., 110, pp. 573-589 (2017).

27. Ranjbar Tezenji, F., Mohammadi, M., Pasandideh, S.H.R., et al. "An integrated model for supplier location-selection and order allocation under capacity constraints in an uncertain environment", Sci. Iran., E, 23(6), pp. 3009-3025 (2016).

28. Jiménez, M. and Bilbao, A. "Pareto-optimal solutions in fuzzy multi-objective linear programming", Fuzzy Set. Syst., 160(18), pp. 2714-2721 (2009).

29. Sakawa, M., Fuzzy Sets and Interactive Multiobjective Optimization, Springer Science \& Business Media (2013).

30. Tiwari, R.N., Dharmar, S., and Rao, J. R. "Fuzzy goal programming-an additive model", Fuzzy Set. Syst., 24(1), pp. 27-34 (1987).

31. Chen, L.H. and Tsai, F.C. "Fuzzy goal programming with different importance and priorities", Eur. J. Oper. Res., 133(3), pp. 548-556 (2001).

32. Wu, Y.K. and Guu, S.M. "A compromise model for solving fuzzy multiple objective linear programming problems", J. Chinese Ins. Indust. Eng., 18(5), pp. 87-93 (2001).

33. Aköz, O. and Petrovic, D. "A fuzzy goal programming method with imprecise goal hierarchy", Eur. J. Oper. Res., 181(3), pp. 1427-1433 (2007).

34. Lee, E.S. and Li, R.J. "Fuzzy multiple objective programming and compromise programming with Pareto optimum", Fuzzy Set. Syst., 53(3), pp. 275-288 (1993).
35. Arikan, F. "A fuzzy solution approach for multi objective supplier selection", Expert Syst. Appl., 40(3), pp. 947-952 (2013).

36. Li, X.Q., Zhang, B., and Li, H. "Computing efficient solutions to fuzzy multiple objective linear programming problems", Fuzzy Set. Syst., 157(10), pp. 13281332 (2006).

37. Erginel, N. and Gecer, A. "Fuzzy multi-objective decision model for calibration supplier selection problem", Comput. Indust. Eng., 102, pp. 166-174 (2016).

38. Govindan, K., Darbari, J.D., Agarwal, V., et al. "Fuzzy multi-objective approach for optimal selection of suppliers and transportation decisions in an ecoefficient closed loop supply chain network", J. Clean. Prod., 165, pp. 1598-1619 (2017).

39. Zadeh, L.A. "Fuzzy sets", Inf. Control, 8(3), pp. 338353 (1965).

40. Alaei, S. and Khoshalhan, F. "A hybrid culturalharmony algorithm for multi-objective supply chain coordination", Sci. Iran. E, 22(3), pp. 1227-1241 (2015).

41. Dubois, D., Kerre, E., Mesiar, R., et al. "Fuzzy interval analysis", In: Dubois, D., Prade, H. (Eds.), Fundamentals of Fuzzy Sets, Kluwer, Massachusetts, pp. 483-561 (2000).

42. Yager, R.R. "Ranking fuzzy subsets over the unit interval", In: Proc. of 17th IEEE Int. Conf. on Dec. Control, San Diego, CA, pp. 1435-1437 (1979).

43. Heilpern, S. "The expected valued of a fuzzy number", Fuzzy Set. Syst., 47, pp. 81-86 (1992).

44. Deb, K., Agrawal, S., Pratap, A., et al. "A fast elitist non-dominated sorting genetic algorithm for multi-objective optimization: NSGA-II", In Int. Conf. on Parallel Prob. Solv. From Nature, pp. 849-858, Springer, Berlin, Heidelberg (2000).

45. Deb, K., Multi-Objective Optimization Using Evolutionary Algorithms, 16, John Wiley \& Sons (2001).

\section{Biographies}

Mohammad Ali Sobhanolahi obtained BSc and MSc degrees in Mathematics from Tabriz University, Tabriz, Iran, in 1973 and 1975, respectively. He also received another $\mathrm{MSc}$ degree in Public Management from Tabriz University, Tabriz, Iran, in 1991 and the $\mathrm{PhD}$ in Industrial Engineering from Swinburne University, Australia, in 1996. Currently, he is a Professor in the Faculty of Industrial Engineering at Kharazmi University, Tehran, Iran. He has authored numerous papers presented at conferences and published in national and international journals.

Ahmad Mahmoodzadeh received BSc degree in Manufacturing Engineering from Shahid Rajaee Teacher Training University, Tehran, Iran, in 2000, and MSc degree in Industrial Engineering from Mazandaran 
University of Science and Technology, Babol, Iran, in 2003. Currently, he is a $\mathrm{PhD}$ candidate in Industrial Engineering at Kharazmi University, Tehran, Iran.

Bahman Naderi completed his BSc degree in Industrial Engineering at Mazandaran University of Science and Technology, Babol, Iran; MSc and PhD degrees in the same field at Amirkabir University of Technology, Tehran, Iran; and post-doctoral program at University of Windsor, Canada. Currently, he is an Assistant Professor at Kharazmi University, Tehran, Iran. His research interest is applied operations research (mathematical modeling and solution method). 